\title{
Microscope-assisted endoscopic interlaminar ligation of spinal arteriovenous fistulas: technical note
}

\author{
${ }^{*}$ Chen Wang, MD, ${ }^{1}$ Chien-Min Chen, MD, PhD, ${ }^{2}$ Fang Shen, MD, PhD, ${ }^{3}$ Xiao-Dong Fang, MD, ${ }^{1}$ \\ Guang-Yu Ying, MD, ${ }^{1}$ Yu-Cheng Ren, MD, ${ }^{1}$ Dan-Feng Yu, MD, ${ }^{1}$ Liang-Liang Zhu, MD, ${ }^{1}$ \\ Yong-Jian Zhu, MD, PhD, ${ }^{1}$ and Jian-Min Zhang, MD, PhD' ${ }^{1}$
}

\begin{abstract}
${ }^{1}$ Department of Neurosurgery, Second Affiliated Hospital of Zhejiang University School of Medicine, Hangzhou; ${ }^{3}$ Department of Neurosurgery, Ningbo No. 2 Hospital, Ningbo, China; and 'Division of Neurosurgery, Department of Surgery, Changhua Christian Hospital, Changhua, Taiwan
\end{abstract}

\begin{abstract}
Spinal dural arteriovenous fistulas (SDAVFs) are the most common type of spinal arteriovenous malformations, and microsurgical ligation is the treatment modality most frequently used for these lesions. Developments in endoscopic techniques have made endoscopy an even less invasive alternative to routine microsurgical approaches in spine surgery, but endoscopic management of SDAVF or other intradural spinal lesions has not been reported to date.

The authors describe the use of a microscope-assisted endoscopic interlaminar approach for the ligation of the proximal draining vein of an L-1 SDAVF in a 58-year-old man. A complete cure was confirmed by postoperative angiography. The postoperative course was uneventful, and short-term follow-up showed improvements in the patient's neurological function. The authors conclude that the endoscopic interlaminar approach with microscope assistance is a safe, minimally invasive, innovative technique for the surgical management of SDAVFs in selected patients.
\end{abstract}

http://thejns.org/doi/abs/10.3171/2015.12.SPINE15366

KEY WORDS endoscope; interlaminar approach; microscope assistance; spinal dural arteriovenous fistula; technique

A LTHOUGH spinal dural arteriovenous fistulas (SDAVFs) are the most common spinal vascular malformations (approximately 70\%), their relative rarity often leads to delay in their diagnosis and treatment. ${ }^{2,21}$ The etiology of SDAVF involves the abnormal development of an arteriovenous shunt between an epidural artery and a dilated pial perimedullary vein, resulting in an escalation of venous congestion and, ultimately, spinal cord edema and ischemic damage..$^{8,11,19}$ Patients with untreated SDAVFs usually present with nonspecific but progressive deterioration of neurological function (e.g., paresthesia, hypesthesia, ataxia, paraplegia, and sphincter disorders). Treatment for SDAVFs includes complete occlusion or ligation of the arteriovenous shunt at the beginning of the draining vein by either endovascular or surgical intervention. ${ }^{16,17,20}$ Although endovascular treatment of SDAVFs is relatively safe, endovascular occlusion of an arteriovenous shunt is not feasible in certain patients due to the variability of spinal cord vascularity, a common origin shared between the feeding artery and the artery of Adamkiewicz, or other technical difficulties such as catheter placement. In addition, the disease recurrence rate associated with endovascular management tends to be higher than that of open surgery., $3,7,10,16$

Interlaminar and transforaminal endoscopic approaches are less invasive than traditional microsurgical approaches for treating various spinal epidural lesions, such as lumbar disc herniation. ${ }^{4,5}$ Recent advances in endoscopic techniques have also significantly expanded their therapeutic indications in spine surgery. ${ }^{14}$ Herein, we report our experience using a microscope-assisted contralateral endoscopic interlaminar approach to repair an SDAVF on the side at the L-1 level. This novel surgical technique was developed for the following reasons. First, the relatively large interlaminar space in the lumbar spine provides a natural "passage" for the insertion of an endoscope. In addition, as the conus medullaris (the lowest level of the spinal cord) ends between the T-12 and L-3 levels in adults, the nerves in the cauda equina continue to branch out and float in the anatomically expanded lumbar canal (lumbar cistern), which provides adequate space for manipulation of endoscopic instruments. Second, the fistulas and the

ABBREVIATIONS DSA = digital subtraction angiography; MRC = Medical Research Council; SDAVF = spinal dural arteriovenous fistula; VRT = volume-rendering tech-

nique.

SUBMITTED April 6, 2015. ACCEPTED December 9, 2015.

INCLUDE WHEN CITING Published online April 15, 2016; DOI: 10.3171/2015.12.SPINE15366.

* Drs. Wang, Chen, and Shen contributed equally to this work. 
draining veins of SDAVFs are usually identified near the intervertebral neural foramina, accompanying the nerve root within its dural sheath. Therefore, during endoscopic surgery, the fistulas, as suggested by preoperative angiography, can be easily identified by tracking the bony landmarks of the spine. Third, the endoscopic imaging system provides a clearer visual field and higher spatial resolution than traditional microsurgical systems, making it feasible to coagulate and cut the fistulas through the endoscopic working channel.

\section{Illustrative Case}

A 58-year-old man presented with a 1-month history of bilateral lower-limb paralysis and numbness and a 2-week history of dysuria. Physical examination revealed no sensation below the level of the anterior superior iliac spine. His muscle strength was Medical Research Council (MRC) Grade 0 in both lower extremities. Magnetic resonance imaging of the spine demonstrated centromedullary T2 signal hyperintensity extending from T-4 to L-1 and perimedullary flow voids along the dorsal and ventral aspect of the thoracolumbar spinal cord (Fig. 1A). Preoperative spinal digital subtraction angiography (DSA) confirmed the lesion to be an SDAVF located at the left L-1 level, which was fed by the root artery descending from the left L-1 intercostal artery (Fig. 1B). After discussion with the patient, an endoscopic interlaminar approach with microscope assistance was chosen as the operative procedure.

\section{Surgical Technique}

After induction of general anesthesia, the patient was placed in a prone position with all pressure points padded. A 4-cm skin incision was made in the midline centered over the T12-L1 interlaminar space (Fig. 2A). Monopolar cauterization was used to divide the right lumbodorsal fascia unilaterally. The interspinous ligament was kept intact. The paraspinous musculature was stripped subperiosteally with monopolar cauterization and, after placement of a retractor, the hemilaminae above and below the T12-L1 interlaminar space and the ligamentum flavum were exposed. Then the microscope was mounted and the unilateral (right) ligamentum flavum was resected piecemeal with a Kerrison rongeur. The dura mater and the arachnoid membrane underneath were then cut open in a standard longitudinal fashion and sutures were placed to tent the dura to form a working window of about 1.5 $\mathrm{cm}$ in diameter. At this point, a rigid endoscope (Joimax foraminoscope for TESSYS) was introduced through the working window and gently inserted into the contralateral (left) subdural space. Isothermal normal saline (at a pressure of $100-150 \mathrm{~mm} \mathrm{H}_{2} \mathrm{O}$ ) was used to irrigate the operative field (see Video 1).

VIDEO 1. After a rigid endoscope was introduced into the contralateral subdural space, the draining vein was identified near the left foramen at the T-12 level. A small piece of gelatin foam was inserted to separate the draining vein from the spinal cord. Distal to the fistula, the draining vein was coagulated by a bipolar probe before sharp dissection of the vein was carried out by a punch.

The stumps of the vessel were examined for hemostasis and the remaining perimedullary veins were found to shrink after ligation. Copyright Yong-Jian Zhu. Published with permission. Click here to view.

The conus medullaris and cauda equina were pulled aside, and the intradural arterialized vein was identified near the left foramen (Fig. 2A). After these intraoperative anatomical findings were checked against preoperative DSA results, the arachnoid membrane between the draining vein and cauda equina was stripped by inserting a
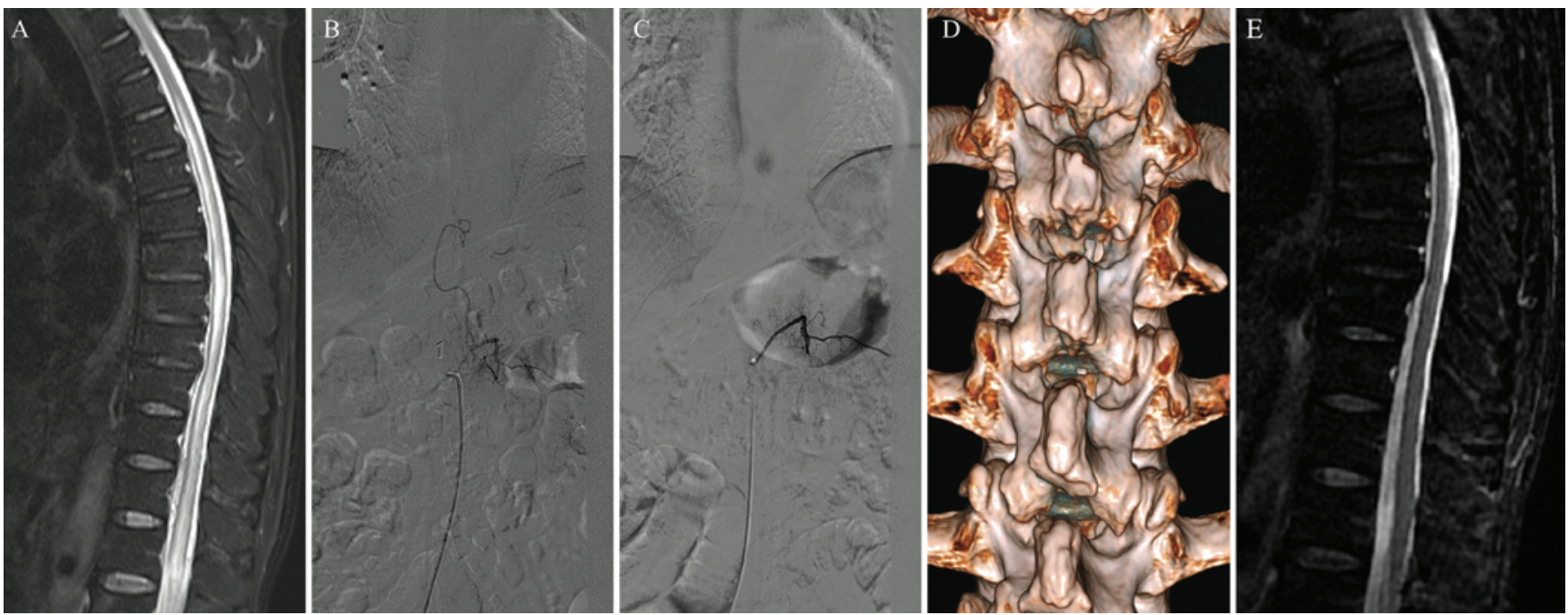

FIG. 1. Radiological findings in the presented case of SDAVF. A: Pretreatment sagittal T2-weighted MR image showing extensive centromedullary edema, increased cord volume, and perimedullary serpiginous small vessels. B: Pretreatment spinal DS angiogram showing the left L-1 intercostal artery feeding the SDAVF and a tortuous draining vein projecting upward. C: Posttreatment angiogram obtained at the same level showing complete occlusion of the fistula. D: Postoperative VRT reconstruction of the lumbar vertebrae shows that the bony structures around the T12-L1 interlaminar space are well preserved after interlaminar neuroendoscopic surgery. E: Follow-up contrast-enhanced T2-weighted MR image obtained 10 months after the operation showing significant decreases in both the centromedullary signal hyperintensity and perimedullary flow void signals in the thoracolumbar spinal cord. Figure is available in color online only. 
small piece of gelatin foam (Fig. 2B). Distal to the fistula, the draining vein was coagulated by means of a bipolar flexible radiofrequency probe (Surgi-Max TM Dual Frequency System), and then sharp dissection of the vein was carried out with a TESSYS endoscopic punch (Fig. 2C). The stumps of the vessel were examined for hemostasis (Fig. 2D), and the remaining perimedullary veins shrank after ligation of the draining vein. At this point, the endoscope was dismounted and the dural wound was closed with surgical titanium clips under microscopic guidance (Fig. 2E). Epidural bleeding was controlled with bipolar cauterization and the fascia was closed with an interrupted absorbable suture. The skin was closed with an interrupted absorbable suture in the deep dermis and with a running absorbable subcuticular stitch. Complete ligation of the fistula was confirmed by postoperative angiography (Fig. 1C). Postoperative volume-rendering technique (VRT) reconstruction of the lumbar vertebrae shows that the bony structures around the T12-L1 interlaminar space were well preserved after interlaminar neuroendoscopic surgery (Fig. 1D). At the clinical evaluation 10 months after the operation, the patient was found to have fully recovered his lower-limb muscle strength (MRC Grade 5 of 5 ) and bowel and bladder sphincter function. The spinal MRI study performed at this follow-up visit showed significant decreases in both the centromedullary $\mathrm{T} 2$ signal hyperintensity and perimedullary flow void signals of the thoracolumbar spinal cord (Fig. 1E).

\section{Discussion}

In the past few decades, neuroendoscopy has become an indispensable technique for treating various neurosurgical conditions, such as intraventricular tumors, skull base tumors, and intracranial cysts..$^{15}$ In spinal surgery, however, the endoscope is still mainly used for spinal epidural conditions like disc herniation, hematoma, and spinal steno- sis. ${ }^{5,22}$ The major reasons hindering the widespread use of spinal endoscopy for intradural spinal lesions are technical difficulties such as opening and closure of the dura mater, the potential for iatrogenic injury to the spinal cord and nerves, and difficulties involved with manipulating instruments through the single endoscopic canal. ${ }^{12}$ Nevertheless, the relatively spacious lumbar spine makes it possible for endoscopic management of intradural lesions through the natural interlaminar space. The endoscopic approach is superior to traditional open microsurgery because it requires a smaller skin incision, leads to better preservation of bony structures of the spine, allows for better illumination of the surgical field and better visualization of anatomical details, and provides better observation angles of lesions. Therefore, endoscopic approaches are increasingly being used to treat other neurosurgical pathologies. ${ }^{5}$

For SDAVFs, both endovascular and microsurgical approaches have been shown to successfully occlude the proximal draining vein and the fistula itself. ${ }^{19,12,13}$ With the help of preoperative spinal DSA, it is generally not difficult to identify the fistula site, the feeding artery, and the drainage pattern. However, the variability of epidural and spinal cord vascular anatomy may still pose diagnostic and therapeutic difficulties for endovascular treatment in some patients. Moreover, the standard microsurgical procedure for SDAVF involves a laminectomy or hemilaminectomy and even partial facetectomy. ${ }^{14,18}$ In this present case, we successfully adopted a much less invasive interlaminar approach to treat lumbar SDAVF. Taking advantage of the natural interlaminar space between T-12 and L-1, the dura was opened under microscopic guidance near the middle line (where the interlaminar space was widest) to form an orifice. This creation of the orifice $(1.5 \mathrm{~cm}$ in diameter) allowed for the insertion of the endoscope toward the contralateral subdural space. Both the irrigation/drainage system and the volume of the lumbar cistern itself allowed for free and safe manipulation of endoscopic surgical instru-
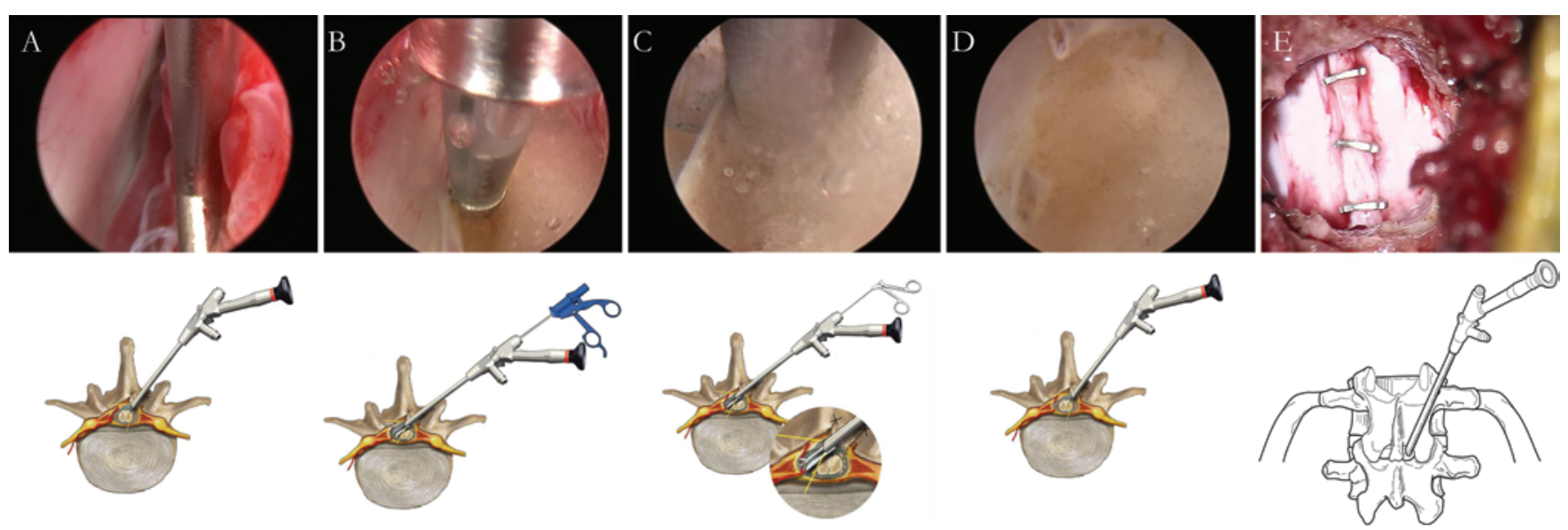

FIG. 2. Intraoperative endoscopic images (upper) and schematic illustrations of the procedure (lower). A: A tortuous vein is seen draining the blood to the surface of the spinal cord through the dura mater. B: The draining vein in the proximity of the fistula was gently separated from the spinal cord, and a small piece of gelatin foam was inserted between the isolated draining vein and the spinal cord. C: The isolated draining vein was cauterized with a bipolar flexible radiofrequency probe and cut off. D: The stumps of the vessel were examined for hemostasis. E: The dural wound was closed with surgical titanium clips under microscopic guidance, and the orifice produced measured about $1.5 \mathrm{~cm}$ in diameter. This tiny opening between the T12-L1 interlaminar space was wide enough for the insertion of the endoscope toward the contralateral subdural space for the occlusion of the lumbar SDAVF. Schematic illustrations: Copyright Wei Zhou. Published with permission. Figure is available in color online only. 
ments. Compared with routine microsurgical ligation of SDAVFs, this novel microscope-assisted endoscopic approach preserves all the bony structures, ensuring reduced risk of postoperative instability and postoperative local pain syndromes in the lumbar spine. To the best of our knowledge, there are no reports of similar surgical techniques for the treatment of SDAVFs.

This interlaminar approach can be used in patients with a clear diagnosis of spinal arteriovenous fistula, plus the location of the fistula has been identified with preoperative DSA. Otherwise, additional openings of the adjacent lamina would be required to expose the lesions. In addition, it would be quite helpful if preoperative CT VRT reconstruction of the lumbar vertebrae was conducted for the determination of the spatial relationship between the fistula and interlaminar space. Further developments in endoscopic technologies ${ }^{15}$ such as chip scopes with flexible tips, 3D neuroendoscopy with LED (light-emitting diode) illumination, and better integration with instruments like microscopes, ultrasonic aspirators, neuronavigation systems, and even robotic-assisted surgical devices, will all contribute to the expansion of the spectrum of neurological pathologies that can be safely and effectively treated by spinal endoscopy. ${ }^{6}$

\section{Conclusions}

The microscope-assisted contralateral endoscopic technique was effective for treatment of SDAVF in our patient, allowing the preservation of bony structures via an interlaminar approach.

\section{Acknowledgments}

We would like to thank Mr. Wei Zhou from the China Academy of Art for his generous help in preparation of the artwork for this manuscript. This study was supported by the Science and Technology Department of Zhejiang Province (Grant No. 2007C33042).

\section{References}

1. Amanieu C, Hermier M, Peyron N, Chabrol A, Deiana G, Manera L: Spinal dural arteriovenous fistula. Diagn Interv Imaging 95:897-902, 2014

2. Chibbaro S, Gory B, Marsella M, Tigan L, Herbrecht A, Orabi M, et al: Surgical management of spinal dural arteriovenous fistulas. J Clin Neurosci 22:180-183, 2015

3. Eneling J, Karlsson PM, Rossitti S: A treatment-refractory spinal dural arteriovenous fistula sharing arterial origin with the artery of Adamkiewicz: Repeated endovascular treatment after failed microsurgery. Surg Neurol Int 5 (Suppl 4):S165-S169, 2014

4. Esposito F, Cappabianca P: Neuroendoscopy: general aspects and principles. World Neurosurg 79 (2 Suppl):S14.e7-S14. e9, 2013

5. Gaab MR: Instrumentation: endoscopes and equipment. World Neurosurg 79 (2 Suppl):S14.e11-S14.e21, 2013

6. Gibson JN, Cowie JG, Iprenburg M: Transforaminal endoscopic spinal surgery: the future 'gold standard' for discectomy? A review. Surgeon 10:290-296, 2012

7. Gokhale S, Khan SA, McDonagh DL, Britz G: Comparison of surgical and endovascular approach in management of spinal dural arteriovenous fistulas: A single center experience of 27 patients. Surg Neurol Int 5:7, 2014

8. Huffmann BC, Gilsbach JM, Thron A: Spinal dural arteriovenous fistulas: a plea for neurosurgical treatment. Acta Neurochir (Wien) 135:44-51, 1995
9. Kirsch M, Berg-Dammer E, Musahl C, Bäzner H, Kühne D, Henkes H: Endovascular management of spinal dural arteriovenous fistulas in 78 patients. Neuroradiology 55:337-343, 2013

10. Lee B, Mehta VA, Mack WJ, Tenser MS, Amar AP: Balloonassisted transarterial embolization of type 1 spinal dural arteriovenous fistula. Neurosurg Focus 37 (1 Suppl):1, 2014

11. Lee TT, Gromelski EB, Bowen BC, Green BA: Diagnostic and surgical management of spinal dural arteriovenous fistulas. Neurosurgery 43:242-247, 1998

12. Marcus J, Schwarz J, Singh IP, Sigounas D, Knopman J, Gobin YP, et al: Spinal dural arteriovenous fistulas: a review. Curr Atheroscler Rep 15:335, 2013

13. Patel NP, Birch BD, Lyons MK, DeMent SE, Elbert GA: Minimally invasive intradural spinal dural arteriovenous fistula ligation. World Neurosurg 80:e267-e270, 2013

14. Patsalides A, Santillan A, Knopman J, Tsiouris AJ, Riina HA, Gobin YP: Endovascular management of spinal dural arteriovenous fistulas. J Neurointerv Surg 3:80-84, 2011

15. Philipps M, Oertel J: High-definition imaging in spinal neuroendoscopy. Minim Invasive Neurosurg 53:142-146, 2010

16. Qi X, Lv L, Han K, Xu Z, Mei Q, Chen H, et al: Analysis of the embolization spinal dural arteriovenous fistula and surgical treatments on 52 cases of the patients. Int J Clin Exp Med 7:3062-3071, 2014

17. Rashad S, Abdel-Bary M, Aziz W, Hassan T: Management of spinal dural arterio-venous fistulas. Report of 12 cases and review of literature. Clin Neurol Neurosurg 125:81-86, 2014

18. Saladino A, Atkinson JL, Rabinstein AA, Piepgras DG, Marsh WR, Krauss WE, et al: Surgical treatment of spinal dural arteriovenous fistulae: a consecutive series of 154 patients. Neurosurgery 67:1350-1358, 2010

19. Tai PA, Tu YK, Liu HM: Surgical treatment of spinal arteriovenous malformations: vascular anatomy and surgical outcome. J Formos Med Assoc 100:389-396, 2001

20. Tuchek CA, Cohen-Gadol AA: Microsurgical ligation of spinal arteriovenous fistulae: techniques. Neurosurg Focus 37 (Suppl 2):Video 11, 2014

21. Yen PP, Ritchie KC, Shankar JJ: Spinal dural arteriovenous fistula: correlation between radiological and clinical findings. J Neurosurg Spine 21:837-842, 2014

22. Zada G, Liu C, Apuzzo ML: "Through the looking glass": optical physics, issues, and the evolution of neuroendoscopy. World Neurosurg 79 (2 Suppl):S3-S13, 2013

\section{Disclosures}

The authors report no conflict of interest concerning the materials or methods used in this study or the findings specified in this paper.

\section{Author Contributions}

Conception and design: YJ Zhu, Wang, Chen, Shen. Acquisition of data: Zhang. Analysis and interpretation of data: Fang, Ying, Ren. Drafting the article: Zhang. Critically revising the article: $\mathrm{Yu}, \mathrm{LL} \mathrm{Zhu}$.

\section{Supplemental Information \\ Videos}

Video 1. https://vimeo.com/150792002.

\section{Correspondence}

Yong-Jian Zhu, Department of Neurosurgery, Second Affiliated Hospital of Zhejiang University School of Medicine, 88 Jiefang Rd., Hangzhou, Zhejiang 310000, China. email: neurosurgery@ zju.edu.cn. 